\title{
Explicitación del conocimiento en pequeñas y medianas empresas. Un análisis desde la perspectiva de redes sociales
}

\author{
PINOCHET SÁNCHEZ, GISELLE \\ Facultad de Ciencias Económicas y Administrativas \\ Universidad Antonio Nariño (Colombia) \\ Correo electrónico: gpinochet@uan.edu.co \\ MARIÑO, JUAN PABLO \\ Facultad de Ciencias Económicas y Administrativas \\ Universidad Antonio Nariño (Colombia) \\ Correo electrónico: jupamarino@uan.edu.co \\ RAMÍREZ TARAZONA, VLADIMIR \\ Facultad de Ciencias Económicas y Administrativas \\ Universidad Antonio Nariño (Colombia) \\ Correo electrónico:vlaramirez@uan.edu.co
}

\begin{abstract}
RESUMEN
Pese a la reconocida importancia sobre la explicitación del conocimiento dentro de la gestión del conocimiento, este proceso ha sido mayormente estudiado desde la perspectiva interna. Es decir, considerando únicamente a los trabajadores como actores de la explicitación, dejando de lado que con frecuencia las fuentes de conocimiento son actores externos. El objetivo de este trabajo es evaluar si existen diferencias significativas entre las empresas que hacen explicitación del conocimiento de forma interna, versus las que incluyen actores externos en este proceso. Para ello, se aplicó un modelo de red en el que los nodos representan actores internos y externos y los vínculos dirigidos dan cuenta de quién es la fuente del conocimiento y quién es quién es responsable de la explicitación del conocimiento. Se aplicó el modelo a 21 pequeñas empresas de servicios colombianas y con base en los resultados obtenidos se encontró que es posible clasificar a las empresas en cuatro categorías según su tendencia a mostrar más o menos explicitación interna, y más o menos explicitación abierta. Los resultados sugieren que existen perfiles de explicitación con mejor desempeño que otros.
\end{abstract}

Palabras clave: explicitación, red, explicitación abierta.

Clasificación JEL: C00; L14; M10.

MSC2010: 03B52; 94C15.

Artículo recibido el 26 de septiembre de 2018 y aceptado el 3 de junio de 2019 


\title{
Explicit knowledge in small and medium enterprises. An analysis from the perspective of social networks
}

\begin{abstract}
Despite of the recognized importance on the explicitation of knowledge within knowledge management, this process has been mainly studied from the internal perspective. That is, considering only the workers as explicit actors, leaving aside that the sources of knowledge are often external actors. The objective of this paper is to evaluate if there are significant differences between companies that make knowledge explicit internally, versus those that include external actors in this process. For this, a network model was applied in which the nodes represent internal and external actors and the directed links give an account of who is the source of knowledge and who is who is responsible for the disclosure of knowledge. The model was applied to 21 small Colombian service companies and based on the results obtained it was found that it is possible to classify companies into four categories according to their tendency to show more or less internal explicitness, and more or less open explicitness. The results suggest that explicitation profiles exist with better performance than others.
\end{abstract}

Keywords: externalization, network, open externalization.

JEL classification: C00; L14; M10.

MSC2010: 03B52; 94C15.

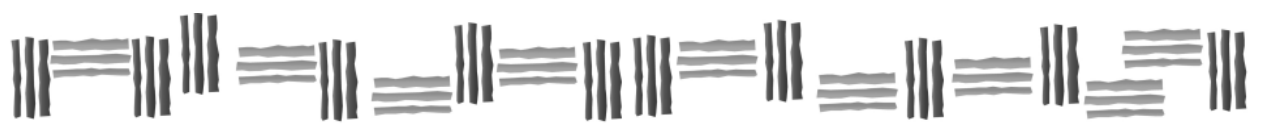




\section{Introducción.}

Diferentes autores coinciden en que la Gestión del Conocimiento (GC) debe concentrar sus esfuerzos en la creación de conocimiento, ya que el nuevo conocimiento es responsable de la mejora del desempeño de la empresa (Nonaka, 2007; Nonaka \& Toyama, 2005). De manera más precisa, se ha afirmado que las diferencias entre los resultados obtenidos por las organizaciones se deben a la mayor creación y explotación del conocimiento (Nonaka, Toyama \& Nagata, 2000).

El conocimiento se crea mediante la transformación del conocimiento, que es el proceso de cambio entre las dos formas de conocimiento que coexisten en la empresa: el conocimiento tácito y el conocimiento explícito. Según el conocimiento origen y el conocimiento que surge como producto de este proceso, pueden distinguirse cuatro tipos de transformación: socialización, que es la transformación de conocimiento tácito a conocimiento tácito; exteriorización, que es la transformación de conocimiento tácito a explícito; la combinación, que es la transformación de conocimiento explícito a conocimiento explícito; e interiorización que es la transformación de conocimiento explícito a conocimiento tácito (Nonaka \& Takeuchi, 1995; Nonaka, 1991).

Aunque todos los procesos de transformación son necesarios para la empresa, se ha señalado que el proceso de explicitación (EC) aporta más valor a la empresa porque crea un activo disponible de manera permanente (Contreras, 2009). En efecto, el conocimiento explícito está libre de limitaciones de tiempo y espacio. Esto permite minimizar el riesgo de que pierda conocimiento vital si las personas clave no están disponibles o abandonan la organización (Nezafati, Afrazeh \& Jalali, 2009; Smith, 2001). Una ventaja adicional de explicitar el conocimiento es que permite identificar deficiencias o vacíos de conocimiento que deben suplirse, lo que consecuentemente motiva la creación de nuevo conocimiento (Nonaka, Toyama \& Konno, 2000).

Sin embargo, los estudios sobre explicitación se han centrado en lo que en este trabajo se denomina explicitación interna del conocimiento (EIC) es decir, explicitación donde el conocimiento proviene exclusivamente de los trabajadores, dejando de lado el hecho de que con frecuencia las ideas para la creación de nuevo conocimiento provienen de fuentes externas a la empresa (González-Sánchez \& García-Muiña, 2011; Kao \& Wu, 2016).

Es por ello que este trabajo tiene como objetivo evaluar si existen diferencias apreciables en el estilo de EC de una muestra de pequeñas y medianas empresas colombianas del sector servicios. Para medir la interacción de los trabajadores en la explicitación del conocimiento (EC) se empleó la ciencia de redes, y cinco medidas de red como indicadores de este proceso. Para evaluar si existen diferencias apreciables entre la explicitación interna del conocimiento (EIC) y la explicitación abierta (EAC) se empleó un algoritmo de agrupamiento difuso. De esta forma, las 21 pequeñas empresas colombianas del sector servicios que conformaron la muestra fueron clasificadas según su tendencia en la EC.

\section{Marco teórico.}

La creación de conocimiento es el proceso de desarrollo de nuevas ideas y soluciones sobre diferentes aspectos de la empresa, como productos, procesos y estrategias de gestión (Nonaka \& Toyama, 2005; Nonaka, 1991). El desarrollo de ventajas competitivas depende en gran medida de la capacidad de las empresas de crear permanentemente nuevo conocimiento, así como de su capacidad de hacerlo extensivo a todas las áreas de la empresa (Navarro \& Olivari, 2016; Aghajani, Yahyazadehfar \& Hosseinzadeh, 2011; Ibarrán, Maffioli \& Stucchi, 2019; Henríquez, 2009; Nonaka, Toyama \& Nagata 2000). Si bien el conocimiento es producto de las capacidades humanas, sin interacción entre el sujeto que crea conocimiento y otros individuos la creación de conocimiento no puede tener lugar (Nonaka \& Takeuchi, 1995; Rai \&Prakash, 2012). Esto hace que la creación de conocimiento sea un proceso inherentemente social, que no se encuentra en la 
individualidad de un actor -interno o externo a la empresa- sino en la relación entre dos o más actores (Nonaka, Toyama \& Konno, 2000; Wei \& Alvarenga, 2010).

El conocimiento organizacional se crea a partir de combinaciones entre el conocimiento tácito y explícito existente en la empresa, a través del proceso de transformación del conocimiento. El conocimiento tácito se caracteriza por ser individual y difícil de formalizar, ya que se pone en práctica sin mediación consciente (Nonaka \& Takeuchi, 1995). Este tipo de conocimiento le permite al individuo resolver problemas a partir de su experiencia y dada su naturaleza informal puede adaptarse a diferentes circunstancias (Muthuveloo, Shanmugam \& Teoh, 2017; Erden, Von Krogh \& Nonaka, 2008). Además, tiene un rol especialmente importante en las tareas grupales porque permite el desarrollo de habilidades del grupo para innovar y afrontar situaciones inesperadas donde no hay reglas o roles predeterminados (Erden, Von Krogh \& Nonaka, 2008; Nonaka \& Toyama, 2007; Rai \& Prakash, 2012). La principal ventaja del conocimiento tácito es que es relativamente económico y fácil de adquirir (Kabir, 2013). No obstante, este conocimiento implica algunos riesgos para las organizaciones, siendo el principal la pérdida frente a la ausencia del trabajador que lo posee (Nezafati, Afrazeh \& Jalali, 2009).

Por su parte, el conocimiento explícito es fácil de comunicar, y pertenece al ámbito de la empresa (Smith, 2001). Puede formalizarse a través de diferentes medios como documentos, bases de datos o procesos. Sin embargo, no debe confundirse cualquier forma de codificación con conocimiento explícito (Contreras, 2009). Para que éste pueda ser considerado como tal debe aportar valor a la empresa y permitir la incorporación de nuevas experiencias. En esencia, el conocimiento explícito no se reduce a la mera reunión de datos sino a su identificación, clasificación y estructuración a través de códigos formales (Nonaka, Toyama \& Konno, 2000). Es por ello que algunos autores han señalado que la explicitación del conocimiento es el proceso de transformación que puede aportarle más ventajas a la empresa (Contreras, 2009; Hemmecke \& Stary, 2004; Herschel, Nemati, \& Steiger, 2001).

La importancia de la explicitación del conocimiento en la empresa indica la necesidad de las empresas de diseñar estrategias para fomentar la creación y uso del conocimiento explícito. $\mathrm{Al}$ respecto, existen diferentes esfuerzos. Por ejemplo, Kinyua, Muathe y Kilika (2015) evaluaron el efecto de los procesos de transformación sobre el desempeño de los procesos en empresas del sector financiero. Aunque este desempeño no contempló indicadores financieros, Aghajani et al. (2011) propusieron un modelo matemático para relacionar los procesos de transformación con la creación de conocimiento. Por su parte, Goonesekera (2012) diseñó un modelo basado en lógica difusa para evaluar la madurez de la gestión del conocimiento, operacionalizado en los cuatro procesos de transformación.

Existen unas condiciones llamadas facilitadores (enablers) que favorecen los cuatro procesos de transformación de conocimiento en la empresa (Nonaka \&Takeuchi, 1995). Las más relevantes son la amplia interacción entre los trabajadores (Takeuchi, 2001), el acceso al conocimiento creado por otros trabajadores (Takeuchi, 2013), la equidad en dicho acceso (Nonaka, Toyama \& Konno, 2000) y la interacción entre los puestos de trabajo (Nonaka \& Konno, 1998). El rol de la empresa es asegurar que existan estas condiciones (Alvarenga \& Choo, 2011; Nonaka, Von Krogh \&Voelpel, 2006).

El carácter social de la EC, evidenciado en las características enunciadas anteriormente, permiten que éste proceso pueda estudiarse y formalizarse desde el análisis de redes sociales (Kao $\& \mathrm{Wu}, 2016$; Wasserman \& Faust, 1994). Una red es un modelo matemático abstracto que permite modelar diferente tipo de sistemas y tiene aplicaciones en diversas áreas del conocimiento como en ciencias de la salud, el transporte y la ingeniería. Una red se define como un conjunto de relaciones entre una serie definida de elementos, y la ciencia de redes es el estudio analítico de dichas redes (Barabási, 2012). Modelar un sistema en forma de red, significa simplificar su realidad en términos de nodos y vínculos, las unidades fundamentales de una red (Barabási, 2012). Para evaluar las propiedades de la red se emplean las medidas de red, indicadores cuantitativos 
del comportamiento de una red (Newman, 2003). La Tabla 1 presenta una muestra de las medidas de red más comúnmente utilizadas; por su parte, la Figura 1 ilustra estas medidas.

Tabla 1. Algunas de las principales medidas de red.

\begin{tabular}{cl}
\hline Medida de red & \multicolumn{1}{c}{ Definición } \\
\hline Centralidad de grado & $\begin{array}{l}\text { Es el número de conexiones que tiene un nodo. En una red } \\
\text { dirigida, puede distinguirse el grado de entrada (vínculos } \\
\text { dirigidos a un nodo) del grado de salida. }\end{array}$ \\
\hline Densidad & $\begin{array}{l}\text { Es la proporción de vínculos que existen en la red sobre el } \\
\text { máximo número posible si todos los nodos estuvieran } \\
\text { conectados entre sí. }\end{array}$ \\
\hline Número de nodos & Es la cantidad de actores de la red. \\
\hline Peso de los vínculos & Es la relevancia del vínculo entre dos nodos. \\
\hline Fuente: Elaboración propia con base en (Barabási, 2012; Rubinov \& Sporns, 2010).
\end{tabular}

Fuente: Elaboración propia con base en (Barabási, 2012; Rubinov \& Sporns, 2010).

Figura 1. Ilustración de una red y algunas de las principales medidas de red.

La red tiene vínculos con
diferentes pesos.
Nodo con mayor centralidad de grado con cuatro nodos.
Un nodo tiene más centralidad de grado porque tiene vínculo
con mayor cantidad de actores.

Fuente: Elaboración propia.

En la literatura sobre ciencia de redes, el término redes de conocimiento se emplea para referirse a las redes que modelan diferentes procesos relacionados con el conocimiento y donde los nodos bien pueden ser individuos, grupos u organizaciones (Phelps, Heidl \&Wadhwa, 2012). Resultados de diferentes estudios de redes de conocimiento permiten concluir que la eficiencia de los procesos relacionados con el conocimiento depende de cómo interactúen los trabajadores en la gestión de dichos procesos (Sutanto, Tan, Battistini \& Phang, 2011; Wang \& Fang, 2011; Singh \& Fleming, 2010; Gao \& Guan, 2009; Schilling \& Phelps, 2007). Por ejemplo, a partir de los estudios de Hahn, Lee y Lee (2015), Eslami, Ebadi \& Schiffauerova (2013) y Yun y Lee (2013) se deduce que el alto grado tiene un efecto positivo sobre la explotación del conocimiento y la innovación, y un efecto negativo sobre la explotación del conocimiento. Adicionalmente, que la homogeneidad entre el grado entre los diferentes nodos demostró un efecto positivo sobre la creación de conocimiento.

También el estudio de Yun y Lee (2013), así como los de Liu, Wang \& Mei (2012), Morrison (2002) y Liu, Fu y Chen (2009) permiten concluir que la alta densidad tiene un efecto positivo sobre la innovación, la transferencia inter e intra organizacional del conocimiento, y sobre la capacidad para desarrollar experticia en las funciones. 
Igualmente, de los estudios de Morrison (2002) y Liu, Fu y Chen (2009) se deriva que un mayor número de nodos en la red tiene un efecto positivo sobre la capacidad de adaptarse la cultura organizacional, y sobre la transferencia intra organizacional del conocimiento. Por último, también de los estudios de Liu, Wang y Mei (2012) y Liu, Fu y Chen (2009) se concluye que vínculos con mayor peso tienen un efecto positivo sobre la transferencia inter e intra organizacional del conocimiento.

\section{Metodología.}

La metodología seguida por este trabajo consiste en tres fases: el diseño del modelo, la recopilación de información y la aplicación preliminar del modelo en una muestra de 21 pequeñas empresas colombianas del sector servicios. A continuación, se describen cada una de las fases.

\section{Modelo de red para evaluar la explicitación del conocimiento}

En la red de explicitación los vínculos representan la creación de un documento gracias a la interacción interna o externa. Los nodos representan personas, parte de la organización o externas a ella, que aportan el conocimiento tácito que es explicitado. La Tabla 2 resume las principales definiciones en las que se basa el modelo de red para evaluar la explicitación abierta.

Tabla 2. Conceptos sobre explicitación del conocimiento y su aplicación al modelo de red.

\begin{tabular}{|c|c|}
\hline Concepto & Aplicación al modelo \\
\hline Conocimiento tácito & $\begin{array}{l}\text { Conocimiento que poseen las personas, internas o externas, que no está } \\
\text { documentado. }\end{array}$ \\
\hline Conocimiento explícito & Conocimiento documentado puesto a disposición de la empresa. \\
\hline Explicitación del conocimiento & Creación de un documento a partir del conocimiento tácito \\
\hline Nodo de la red de explicitación & $\begin{array}{l}\text { Persona participa en la explicitación de conocimiento, como fuente de } \\
\text { conocimiento tácito o como actor que explicita el conocimiento. }\end{array}$ \\
\hline Nodo interno & Trabajador de la empresa. \\
\hline Nodo externo & Fuente externa de conocimiento tácito. \\
\hline $\begin{array}{l}\text { Vínculo de la red de explicitación } \\
\text { Vínculo interno } \\
\text { Vínculo externo }\end{array}$ & $\begin{array}{l}\text { Relación de la cual se extrae conocimiento tácito que luego es explicitado. } \\
\text { Vínculo que conecta dos nodos internos. } \\
\text { Vínculo que conecta un nodo interno con un nodo externo. }\end{array}$ \\
\hline
\end{tabular}

En la red de explicitación abierta los vínculos tienen una dirección que implica ser fuente de conocimiento tácito como lo muestra la ecuación (1):

$$
l_{I J}
$$

donde $l$ es un vínculo entre los nodos $i$ y $j$, y donde $j$ es fuente de conocimiento tácito del nodo $i$. Se establece un vínculo entre los nodos si el nodo $i$ reporta haber creado al menos un documento gracias al conocimiento obtenido de $j$. Los vínculos tienen peso igual a 1 .

Para cuantificar la EC se emplean cuatro indicadores, como se muestra en la Tabla 3. 
Tabla 3. Indicadores de medición de la explicitación abierta.

Indicador

Centralización de grado de entrada

Centralidad de grado de salida

Densidad interna

Cantidad de explicitación externa
Aplicación al modelo

Evalúa la tendencia de la red a evidenciar unos pocos nodos que fungen como fuente de conocimiento tácito para otros muchos nodos.

Evalúa la tendencia de la red a evidenciar unos pocos nodos han participado en numerosos procesos de explicitación del conocimiento.

Evalúa la densidad entre los nodos internos.

Evalúa la proporción entre la cantidad de nodos externa y el número total de nodos.

Fuente: Elaboración propia.

Respecto a la densidad, es necesario precisar que se mide la densidad entre los nodos internos para evaluar la EC general de la empresa, ya que la densidad general de la red (considerando nodos internos y externos) nunca será alta. Esto se debe a que es poco probable que una empresa tenga más fuentes externas que internas, aunque pueda encontrarse algún caso aislado en micro o pequeñas. Por lo tanto, la densidad general no será un indicador apropiado que permita discriminar a las empresas según su cantidad de EC. En relación con esta medida es importante precisar que la densidad mide la proporción de vínculos existentes sobre vínculos posibles, sin discriminar el sentido del vínculo.

Por el contrario, la cantidad de explicitación externa mide si la mayor cantidad de fuentes de explicitación provienen del exterior o no. Pero esta medida no es capaz por sí misma de evaluar si una empresa lleva a cabo procesos de EC, y por ello ambas medidas son necesarias y complementarias para modelar adecuadamente la complejidad del proceso de EC.

\section{Recopilación de información}

Se evaluaron 21 pequeñas empresas del sector servicios, que se distribuyen como se muestra en la Figura 2.

Figura 2. Distribución de la muestra de empresas según actividad y número de puestos de trabajo.

Distribución de empresas según actividad.

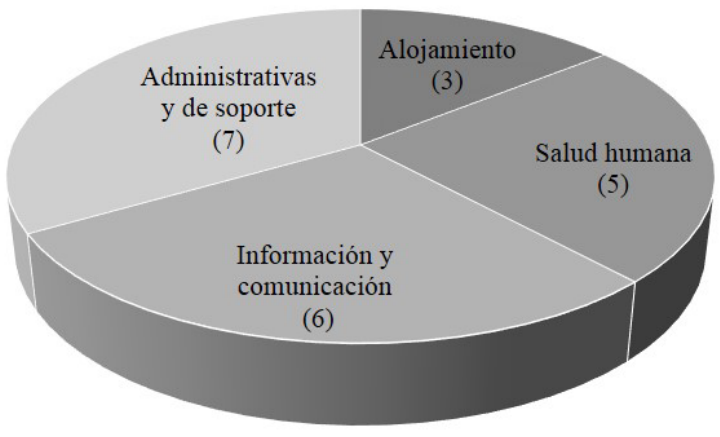

Número de puestos de trabajo por empresa.

\begin{tabular}{|l|l|}
\hline $\mathrm{e} 1$ & 23 \\
\hline $\mathrm{e} 2$ & 22 \\
\hline $\mathrm{e} 3$ & 19 \\
\hline $\mathrm{e} 4$ & 20 \\
\hline $\mathrm{e} 5$ & 17 \\
\hline $\mathrm{e} 6$ & 18 \\
\hline $\mathrm{e} 7$ & 18 \\
\hline $\mathrm{e} 8$ & 20 \\
\hline $\mathrm{e} 9$ & 16 \\
\hline $\mathrm{e} 10$ & 14 \\
\hline $\mathrm{e} 11$ & 22 \\
\hline
\end{tabular}

\begin{tabular}{|l|l|}
\hline $\mathrm{e} 12$ & 18 \\
\hline $\mathrm{e} 13$ & 21 \\
\hline $\mathrm{e} 14$ & 16 \\
\hline $\mathrm{e} 15$ & 16 \\
\hline $\mathrm{e} 16$ & 20 \\
\hline $\mathrm{e} 17$ & 17 \\
\hline $\mathrm{e} 18$ & 15 \\
\hline $\mathrm{e} 19$ & 20 \\
\hline $\mathrm{e} 20$ & 18 \\
\hline $\mathrm{e} 21$ & 20 \\
\hline
\end{tabular}

Fuente: Elaboración propia. 
La información necesaria para aplicar el modelo es número de documentos en los que participó cada trabajador y fuentes de conocimiento tácito a partir de los que se crearon dichos documentos. De acuerdo con la Tabla 2, las fuentes pueden ser internas o externas. La información se obtuvo a través de un formulario en línea, que se reproduce en la Tabla 4. Adicionalmente, a las empresas que aceptaron participar en el estudio se les pidió un listado de la totalidad de puestos de trabajo de la organización.

Tabla 4. Formato para la recopilación de información.

\begin{tabular}{ll}
\hline 1 & Nombre de la empresa donde trabaja \\
\hline 2 & Cargo \\
\hline 3 & $\begin{array}{l}\text { ¿Ha participado en la creación y puesta a disposición de la empresa de uno o más documentos? } \\
\text { (Responda Sí o No) }\end{array}$ \\
\hline 4 & $\begin{array}{l}\text { En caso de haber respondido afirmativamente la pregunta 3, por favor indique qué cargos de } \\
\text { la empresa contribuyeron con información, sugerencias o algún otro aporte en la elaboración } \\
\text { de dichos documentos. }\end{array}$ \\
\hline 5 & $\begin{array}{l}\text { En caso de haber respondido afirmativamente la pregunta 3, por favor indique qué personas } \\
\text { externas a la empresa contribuyeron con información, sugerencias o algún otro aporte en la } \\
\text { elaboración de dichos documentos. }\end{array}$ \\
\hline
\end{tabular}

Fuente: Elaboración propia.

\section{Aplicación del modelo}

Con la información obtenida se creó una red para cada empresa de la muestra en la que cada nodo representa un actor en la explicitación del conocimiento y cada vínculo representa una contribución en dicha explicitación. Las redes se crearon empleando el programa Gephi el cual permite generar tanto la representación gráfica de la red como las medidas de red.

Para clasificar a las empresas según su tendencia de EC se empleó algoritmo de agrupamiento difuso fuzzy $c$-means, en el que cada elemento pertenece a todos los conjuntos y la pertenencia a cada conjunto de cada elemento se mide por un coeficiente de pertenencia que toma valores entre 0 y 1 , donde 0 indica la no pertenencia y el 1 indica la pertenencia total. La suma de los coeficientes de pertenencia de un elemento a todos los conjuntos es de 1, indicando que cada elemento debe pertenecer completamente a los conjuntos posibles, independientemente de su pertenencia parcial a cada uno.

Para calcular la pertenencia de un elemento a un conjunto se utiliza la distancia euclidiana del elemento al centroide del conjunto. El centroide es un elemento ficticio que representa las características típicas del conjunto y se localiza en un punto donde la suma de las distancias de todos los elementos del conjunto al centroide es mínima (Gonzalez \& Barato, 2003). Un elemento presentará mayor pertenencia a un conjunto si su distancia al centroide de ese conjunto es menor (Sosa-García, Vega-Pons \& Ruiz-Shulcloper, 2012). Esta cercanía permite calcular el coeficiente de pertenencia de cada elemento al conjunto. Para afirmar que una empresa pertenece inequívocamente a uno de los dos conjuntos se estableció un umbral de 0,8 en el coeficiente de pertenencia.

Como se mencionó previamente, la cantidad de explicitación externa mide a dónde pertenece la mayor cantidad de fuentes (dentro o fuera de la empresa). Pero esta información es insuficiente para medir la cantidad general de EC de la empresa, lo que se evalúa mediante la densidad interna y, por tanto, ambas medidas fueron empleadas en el agrupamiento. Para afirmar que las empresas de la muestra pueden clasificarse según sus redes de EC en EIC o EAC y según su nivel general de EC, la totalidad de las empresas o una parte significativa de la muestra debe pertenecer a uno de los dos conjuntos.

Por último, se evaluó también la centralización de grado. La alta centralización de grado de entrada supone que unas pocas personas son fuente de conocimiento tácito de una empresa 
determinada. Mientras que una alta centralización de grado de salida implica que unas pocas personas son creadores de conocimiento explícito.

\section{Resultados.}

Después de construir las redes de EC para cada una de las empresas de la muestra se calculó cantidad de explicitación externa y de densidad interna; los resultados se presentan en la Figura 3.

Figura 3. Cantidad de explicitación externa y densidad interna por empresa.

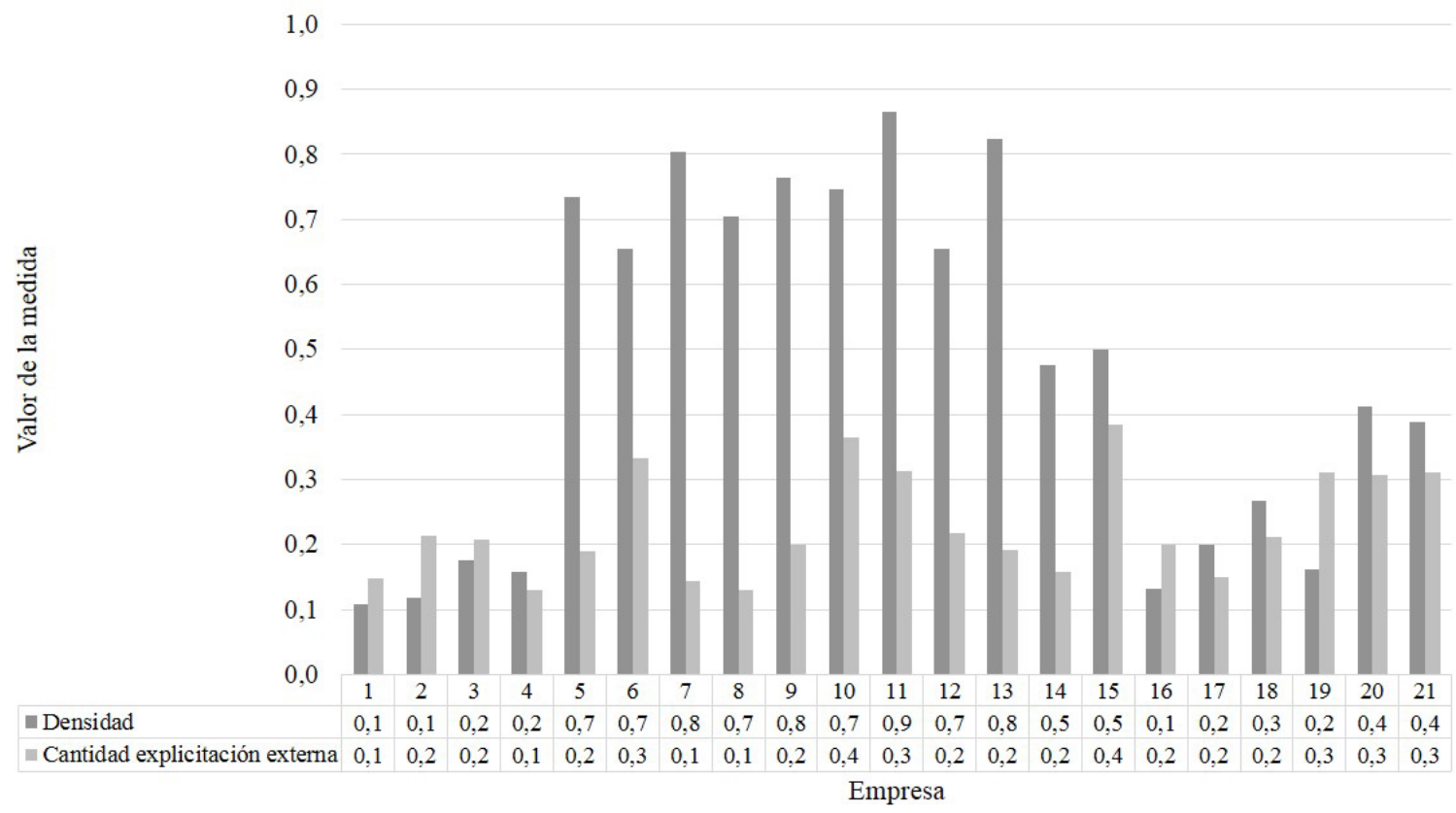

Fuente: Elaboración propia.

Posteriormente, se aplicó el agrupamiento difuso. La Tabla 5 muestra para ambas medidas los valores del centroide, que es el valor representativo en cada uno de los conjuntos. Adicionalmente, se muestra el promedio de las distancias entre el valor de esta medida para cada empresa y el valor del centroide.

Tabla 5. Valores del centroide para los conjuntos EAC y EIC y distancias entre cada elemento al centroide.

\begin{tabular}{|c|c|c|c|}
\hline Agrupación & & Valor del centroide & Promedio de las distancias al centroide \\
\hline \multirow[b]{2}{*}{$\mathrm{EC}$} & EAC & 0,15 & 0,003 \\
\hline & EIC & 0,34 & 0,001 \\
\hline \multirow[b]{2}{*}{ Densidad interna } & Alta & 0,26 & 0,005 \\
\hline & Baja & 0,75 & 0,0003 \\
\hline
\end{tabular}

Fuente: Elaboración propia.

Los resultados de la Tabla 5 muestran que en ambos casos el promedio de distancias al centroide es muy reducido. Esto indica que no hay diferencias significativas entre los valores de esta medida para las empresas que pertenecen a uno y otro conjunto y, por tanto, cada conjunto y su respectivo centroide las representa adecuadamente. 
Tras evaluar las dos medidas mencionadas anteriormente, se evaluó la centralización de grado de entrada y grado de salida por cada empresa. La Tabla 7 muestra el nivel de EC y de centralización para cada empresa. Es importante señalar que el tipo de EC que se presenta en esta tabla hace referencia a cuál de los dos perfiles (EIC o EAC) tiene mayor prevalencia sobre el otro, en valor absoluto. Sin embargo, no implica necesariamente que la empresa tenga alto nivel de EC.

Tabla 7. Clasificación de cada empresa de la muestra según nivel de densidad interna, tipo de EC y centralización.

\begin{tabular}{|c|c|c|c|c|c|c|c|c|c|}
\hline Empresa & $\begin{array}{c}\text { Nivel } \\
\text { densidad } \\
\text { interna }\end{array}$ & $\begin{array}{c}\text { Tipo } \\
\text { EC }\end{array}$ & $\mathrm{Ce}^{*}$ & $\mathrm{Cs}^{* *}$ & Empresa & $\begin{array}{c}\text { Nivel } \\
\text { densidad } \\
\text { interna } \\
\end{array}$ & $\begin{array}{c}\text { Tipo } \\
\text { EC }\end{array}$ & $\mathrm{Ce}^{*}$ & $\mathrm{Cs}^{* *}$ \\
\hline 1 & Baja & EIC & Alta & Alta & 12 & Alta & EIC & Baja & Baja \\
\hline 2 & Baja & EIC & Alta & Alta & 13 & Alta & EIC & Baja & Baja \\
\hline 3 & Baja & EIC & Alta & Baja & 14 & Baja & EIC & Baja & Baja \\
\hline 4 & Baja & EAC & Alta & Baja & 15 & Baja & EAC & Baja & Baja \\
\hline 5 & Alta & EIC & Baja & Baja & 16 & Baja & EIC & Alta & Baja \\
\hline 6 & Alta & EAC & Baja & Baja & 17 & Baja & EIC & Alta & Baja \\
\hline 7 & Alta & EIC & Baja & Baja & 18 & Baja & EIC & Alta & Baja \\
\hline 8 & Alta & EIC & Baja & Baja & 19 & Baja & EAC & Alta & Baja \\
\hline 9 & Alta & EIC & Baja & Baja & 20 & Baja & EAC & Baja & Baja \\
\hline 10 & Alta & EAC & Baja & Baja & 21 & Baja & EAC & Baja & Baja \\
\hline 11 & Alta & EAC & Baja & Baja & & & & & \\
\hline
\end{tabular}

* Centralización grado de entrada

** Centralización grado de salida

Fuente: Elaboración propia.

A partir de los resultados del agrupamiento según densidad interna y cantidad de explicitación externa, se propone que las empresas pueden clasificarse empleando un sistema que combina dos variables, donde cada una de ellas puede tener un nivel alto o bajo, lo que da lugar a cuatro combinaciones posibles como se muestra en la Figura 4. 
Figura 4. Clasificación de empresas según el nivel de EC interno y externo.

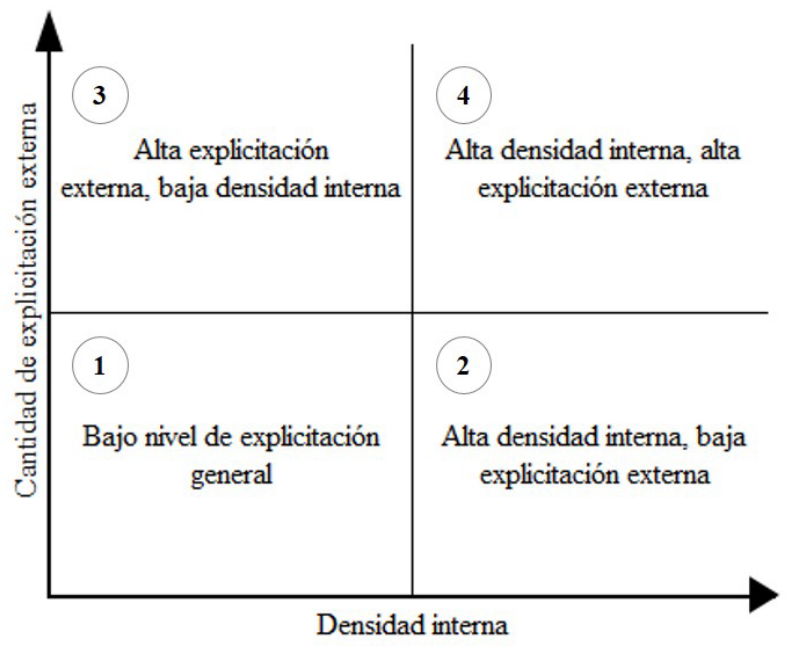

Fuente: Elaboración propia.

Un ejemplo de empresa de cada uno de los cuadrantes se presenta en la Figura 5.

Figura 5. Ejemplo de empresas de cada cuadrante de EC.

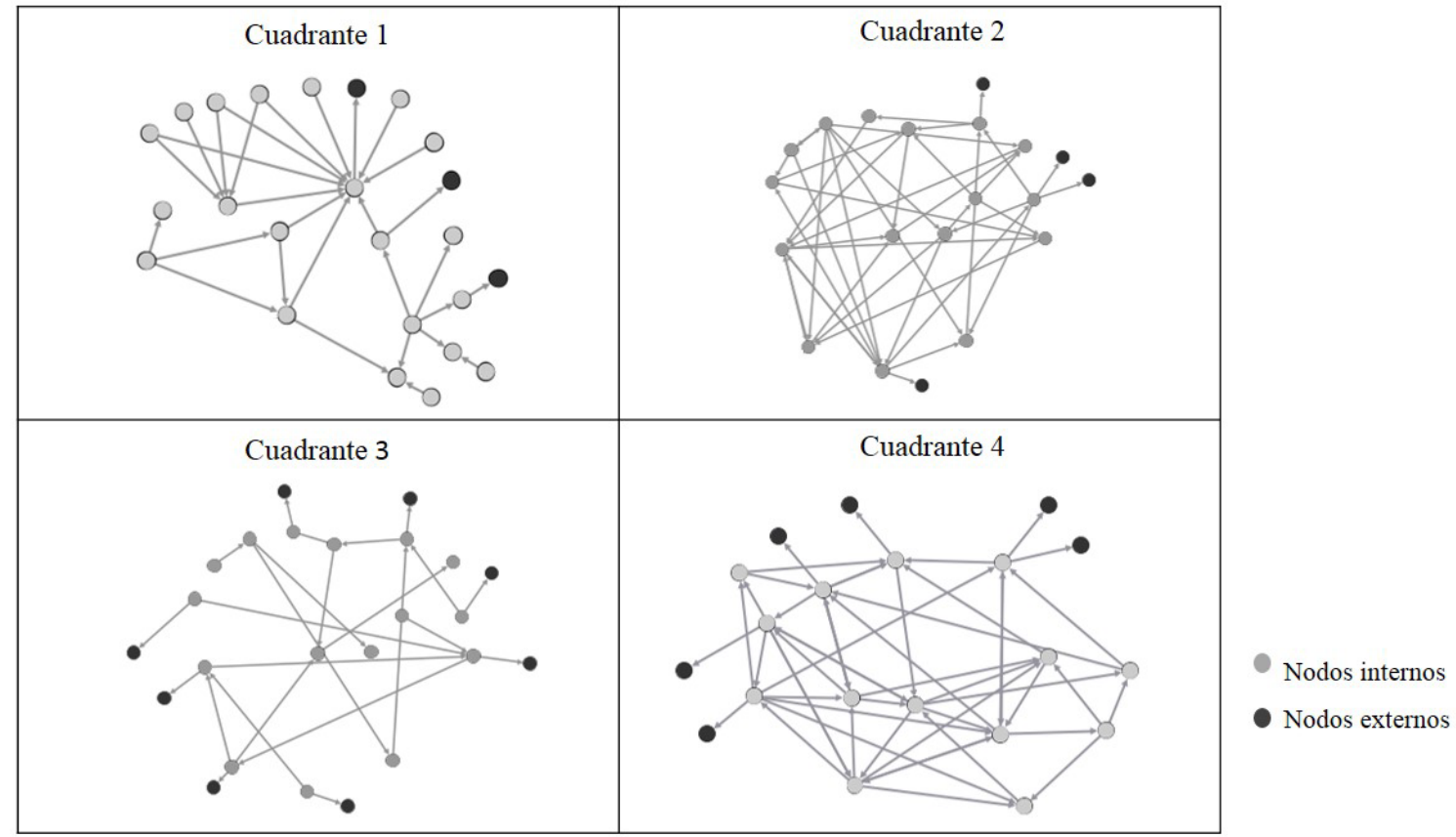

Fuente: Elaboración propia.

\section{Discusiones y conclusiones.}

Los resultados de la Figura 3 muestran que, siendo que las medidas densidad interna y cantidad de explicitación externa pueden tomar valores en el rango entre 0 y 1 , en la mayoría de casos (15 de 21 empresas) la densidad interna es superior a la cantidad de explicitación externa. Y en los casos en que la cantidad de explicitación externa es superior es porque la densidad es significativamente baja (inferior a 0,2). Esto podría explicarse por el hecho de que los colaboradores son en sí mismos un recurso suficientemente valioso para explicitar conocimiento, por lo que podría no justificarse adecuadamente -considerando la relación costo beneficio- de acudir a fuentes externas. Sin embargo, también podría representar una limitación en los procesos de gestión del conocimiento de estas empresas al no identificar adecuadamente todas las fuentes de conocimiento disponibles de la empresa. Especialmente, si se considera que los trabajadores 
pueden establecer contactos externos como parte de sus responsabilidades, por lo que no supone una inversión de recursos adicionales para la empresa.

Los resultados del agrupamiento difuso mostraron que todas las empresas tuvieron una clara pertenencia a uno de los clusters, representado en un coeficiente de pertenencia igual o superior a 0,8 . Estos resultados confirman que las empresas pueden distinguirse según su estilo de EC. Una empresa típica de EIC es aquella en donde un 15\% de las fuentes de conocimiento son internas, mientras que una empresa típica de EAC es aquella donde el $34 \%$ de las fuentes de conocimiento provienen del exterior. En términos absolutos, un 34\% podría considerarse escaso. Pero en términos relativos puede suponer una importante cantidad de esfuerzo para la empresa lograr que más del $30 \%$ de sus fuentes de conocimiento tácito provengan del exterior. Y más aún implementar estrategias para identificar conocimiento tácito externo y convertirlo en conocimiento explícito de la empresa. De manera complementaria, una típica empresa con baja EC tiene una densidad interna de 0,26 y una típica empresa con alta EC tiene una densidad externa de 0,75 . En contraste con la explicitación externa, una empresa con alta EAC tiene el $75 \%$ de posibles vínculos, un valor significativamente superior al 34\% que representa una EIC alto.

Al combinar ambas variables, se tiene un sistema de clasificación de empresas en cuatro categorías de EC. Esta propuesta permite evaluar a las empresas según su perfil de EC y establecer estrategias de mejora a partir de dicho estado. Las empresas del cuadrante 1 son aquellas que tienen un bajo nivel de EC general por lo que no se discrimina si el mayor número de fuentes de conocimiento tácito son internas o externas. Estas empresas se caracterizan por un escaso fomento a la EC y podrían, por tanto, verse privadas de los beneficios que supone documentar y poner a disposición de la empresa el conocimiento que poseen los trabajadores. Las empresas del cuadrante 2 generan un alto número de procesos de EC y la mayoría de sus fuentes son internas, siendo lo opuesto a las empresas del cuadrante 3. Por último, las empresas del cuadrante 4 poseen alto número de procesos de $\mathrm{EC}$, con fuentes internas y externas.

La Figura 5 muestra que algunas de las diferencias entre las empresas de cada cuadrante de EC pueden apreciarse a simple vista. Las empresas del cuadrante 1 y 3 se asemejan en tener una escasa densidad interna, pero se diferencian en que la proporción de nodos externos versus nodos internos es significativamente mayor en las empresas del cuadrante 3. Por el contrario, las empresas del cuadrante 2 y 4 se asemejan en una alta densidad interna, pero se diferencian en que la empresa del cuadrante 4 tiene mayor proporción de nodos externos. De manera complementaria, las medidas de red permiten cuantificar con precisión las diferencias estructurales entre las empresas aportando así información adicional a la que puede percibirse gracias a la representación gráfica de las redes.

Estructuralmente, las redes muestran unas características de EC bastantes diferentes, evaluadas con dos rasgos de la EC: la cantidad de interacción entre los trabajadores (densidad interna) y por la cantidad de fuentes externas de conocimiento (cantidad de explicitación externa). A partir de la literatura revisada sobre facilitadores de la transformación del conocimiento, las empresas del cuadrante 4 son las que presentan mejor desempeño en EC porque fomentan tanto la participación de sus trabajadores como la inclusión del conocimiento externo en la creación de conocimiento documentado. Por el contrario, a la luz de dichos criterios, las empresas del cuadrante 1 presentan un nivel inferior en el desempeño de los procesos de EC.

Las empresas de los cuadrantes 2 y 3 se encuentran en niveles intermedios pero la teoría no ofrece suficientes elementos conceptuales para sostener que uno de estos perfiles suponga un mejor desempeño. Por un lado, se podría afirmar que la densidad interna se relaciona con la amplia interacción entre los trabajadores y por esta razón las empresas del cuadrante 2 tendrían una mejor gestión de los procesos de EC. Sin embargo, estas mismas empresas estarían explotando inadecuadamente sus fuentes externas, muchas de ellas existentes sin necesidad de hacer mayores inversiones. 
En relación con la centralización de las redes, se observa que la mayoría de empresas con baja densidad interna presentan alta centralización de entrada (10 de 12 empresas con baja densidad interna). Por el contrario, todas las empresas que tuvieron alta densidad interna tuvieron baja centralización de entrada. Podría, por tanto, plantearse la hipótesis de que existe alguna relación entre pertenecer al cuadrante 1 y el escaso aprovechamiento de las fuentes de conocimiento. Es interesante notar que esto no se equipara con una escasa cantidad de fuentes de conocimiento. Como señalaba Takeuchi (2013), todos los trabajadores tienen el potencial para transformar conocimiento. Es responsabilidad de la empresa crear las condiciones necesarias para que ese potencial se vuelva realidad.

El probable fenómeno de asociación entre densidad interna y centralización no parece extenderse a la centralización de salida. Esto implicaría que no existe relación entre la escasa cantidad de EC y la tendencia a que unos pocos trabajadores sean responsables de la transformación del conocimiento. Una posible explicación a esta situación es que es más probable que exista menos explotación de fuentes de conocimiento que capacidad de creación de conocimiento. En otras palabras, que la responsabilidad de la escasa EC radicaría en la falta de aprovechamiento del conocimiento.

Las empresas son sistemas complejos por la diversidad de factores internos y externos e interacciones entre ellas; y especialmente complejas en lo que se refiere a procesos relacionados con el uso y explotación del conocimiento. Es por ello que necesitan ser representadas por modelos que capturen esta complejidad con más de una variable. Por lo tanto, aprovechando la capacidad de la ciencia de redes para representar estructuras sociales complejas, el modelo desarrollado en este trabajo necesita por lo menos dos medidas de red. Otros autores han tenido éxito al emplear la ciencia de redes como herramienta para evaluar aspectos relacionados con el conocimiento (Yun \& Lee, 2013; Hahn, Lee \& Lee, 2015), coincidiendo en que la realidad de la estructura se ve reflejada en las medidas de red. De esta forma, las medidas se convierten en indicadores cuantitativos de su objeto de estudio. De manera preliminar, los resultados de la aplicación del modelo son coherentes con la utilidad de la densidad y la centralidad para evaluar procesos relacionados con el conocimiento.

En este trabajo no se incluyó el peso de los vínculos lo que no permite evaluar el efecto de la relevancia de un proceso de EC, o la cantidad de estos procesos, sobre el perfil de EC de la empresa. Otra limitación del estudio es que no se contraste el perfil de EC con un indicador externo y objetivo de desempeño de la empresa, lo que no permite evaluar si dicho perfil tiene un impacto sobre los resultados organizacionales. Por último, los resultados obtenidos sugieren que sería conveniente evaluar si existe correlación fuerte y significativa entre la densidad interna y la centralización de grado de salida. De esta forma sería posible conocer si la escasa cantidad de EC se asocia con la dificultad para aprovechar las fuentes de conocimiento disponibles.

Estas propuestas quedan por comprobarse cuantitativamente en futuros trabajos, ya que sus resultados pueden significar grandes impactos sobre la mejora del aprovechamiento del conocimiento en las empresas.

Aunque el modelo de evaluación de la EC presentado en este estudio ha sido aplicado sólo en pequeñas empresas, los aspectos de cuantificación objetiva son válidos para cualquier tipo de organización. En concordancia con lo anterior, una futura línea de investigación debe ser la aplicación del modelo a empresas con mayor tamaño para observar su comportamiento y evaluar la consistencia de los tipos de empresa según su estructura EC. 


\section{Referencias}

Aghajani, H., Yahyazadehfar, M., \& Hosseinzadeh, S.A. (2011). Knowledge creation processes: a survey of SMEs in the Iranian Province of Mazandaran. Journal of Global Entrepreneurship Research, 1(1), 59-71.

Alvarenga, R.C.D.D., \& Choo, C.W. (2011). Expanding the concept of Ba: managing enabling contexts in knowledge organizations. Perspectivas em Ciência da Informação, 16(3), 225 .

Barabási, A, L, (2012). Linked: The new science of networks. New York: Plume.

Contreras, E. (2009). Transformación de Conocimiento Tácito en Explícito, una revisión crítica. Santiago de Chile, Chile: Ceges.

Erden, Z., Von Krogh, G., \& Nonaka, I. (2008). The quality of group tacit knowledge. The Journal of Strategic Information Systems, 17(1), 4-18. DOI: 10.1016/j.jsis.2008.02.002.

Eslami, H., Ebadi, A., \& Schiffauerova, A. (2013). Effect of collaboration network structure on knowledge creation and technological performance: the case of biotechnology in Canada. Scientometrics, 97(1), 99-119. DOI: 10.1007/s11192-013-1069-6.

Gao, X., \& Guan, J. (2009). Networks of scientific journals: An exploration of Chinese patent data. Scientometrics, 80(1), 283-302. DOI: 10.1007/s11192-007-2013-4

González, D. \& Barato, S. (2003). Modelamiento difuso con técnicas de Clustering. Ingeniería, 8(1), 86-94.

González-Sánchez, R., \& García-Muiña, F. E. (2011). Innovación abierta: Un modelo preliminar desde la gestión del conocimiento. Intangible capital,7(1), 82-115. DOI: 10.3926/ic.2011.v7n1.p82-115.

Goonesekera, T. (2012). Measuring Knowledge Management Maturity Levels in the Manufacturing Sector Using Fuzzy Logic Theory (Doctoral dissertation, La Trobe University).

Hahn, M.H., Lee, K.C., \& Lee, D.S. (2015). Network structure, organizational learning culture, and employee creativity in system integration companies: the mediating effects of exploitation and exploration. Computers in Human Behavior, 42, 167-175. DOI: 10.1016/j.chb.2013.10.026.

Hemmecke, J., \& Stary, C. (2004, April). A framework for the externalization of tacit knowledge embedding repertory grids. In Proceedings OKLC-2004, 5th European Conference on Organizational Knowledge, Learning and Capabilities, Innsbruck (Vol. 56).

Henriquez, L. (2009). Políticas para las Mipymes frente a la crisis. Conclusiones de un estudio comparativo de América Latina y Europa. Documento de Trabajo Europe Aid y ONUDI.

Herschel, R.T., Nemati, H., \& Steiger, D. (2001). Tacit to explicit knowledge conversion: knowledge exchange protocols. Journal of Knowledge Management, 5(1), 107-116. DOI: $10.1108 / 13673270110384455$. 
Ibarrarán, P., Maffioli, A., \& Stucchi, R. (2009). SME policy and firms' productivity in Latin America.

Kabir, N. (2013). Tacit knowledge, its codification and technological advancement. Electronic Journal of Knowledge Management, 11(3), 235-243.

Kao, S.C., \& Wu, C. (2016). The role of creation mode and social networking mode in knowledge creation performance: Mediation effect of creation process. Information \& Management, 53(6), 803-816. DOI: 10.1016/j.im.2016.03.002.

Kinyua, G.M., Muathe, S.M.A., \& Kilika, J.M. (2015). Effect of knowledge conversion and knowledge application on performance of commercial banks in Kenya. International Journal of Education and Research, 3(10), 431-445.

Liu, H., Fu, Y., \& Chen, Z. (2009). Effects of social network on knowledge transfer within R\&D team. In Information Management, Innovation Management and Industrial Engineering, 2009 International Conference on (Vol. 3, pp. 158-162). IEEE.

Liu, H., Wang, Q., \& Mei, Y. (2012). Effects of start-up firm's social network on knowledge transfer: An empirical study. In Information Management, Innovation Management and Industrial Engineering (ICIII), 2012 International Conference on (Vol. 3, pp. 228-233). IEEE.

Morrison, E. (2002). Newcomers'relationship: the role of social network ties during socialization. Academy of Mangement Journal, 45, 1149-1160. DOI: 10.5465/3069430.

Muthuveloo, R., Shanmugam, N., \& Teoh, A.P. (2017). The impact of tacit knowledge management on organizational performance: Evidence from Malaysia. Asia Pacific Management Review, 22(4), 192-201. DOI: 10.1016/j.apmrv.2017.07.010.

Navarro, J.C., \& Olivari, J. (2016). La política de innovación en América Latina y el Caribe. Nuevos caminos. Washington DCB: Banco Interamericano de Desarrollo.

Newman, M.E. (2003). The structure and function of complex networks. SIAM review, 45(2), $167-256$

Nezafati, N., Afrazeh, A., \& Jalali, S.M.J. (2009). A dynamic model for measuring knowledge level of organizations based on Nonaka and Takeuchi Model (SECI). Scientific Research and Essays, 4(5), 531-542.

Nonaka, I. (1991). The knowledge creating company. Harvard Business Review, 69, 96-104.

Nonaka, I., \& Takeuchi, H. (1995). La organización creadora de conocimiento. Cómo las compañías japonesas crean la dinámica de la innovación (M.H. Kocka, Trans. $1^{\mathrm{a}}$ ed.). México: Oxford University Press.

Nonaka, I., \& Konno, N. (1998). The concept of "Ba": Building a foundation for knowledge creation. California Management Review, 40(3), 40-54.

Nonaka, I., Toyama, R., \& Konno, N. (2000). SECI, Ba and leadership: a unified model of dynamic knowledge creation. Long Range Planning, 33(1), 5-34. DOI: 10.1016/S00246301(99)00115-6. 
Nonaka, I., Toyama, R., \& Nagata, A. (2000). A firm as a knowledge-creating entity: a new perspective on the theory of the firm. Industrial and corporate change, 9(1), 1-20. DOI: 10.1093/icc/9.1.1.

Nonaka, I., \& Toyama, R. (2005). The theory of the knowledge-creating firm: subjectivity, objectivity and synthesis. Industrial and Corporate Change, 14(3), 419-436. DOI: 10.1093/icc/dth058.

Nonaka, I., Von Krogh, G., \& Voelpel, S. (2006). Organizational knowledge creation theory: Evolutionary paths and future advances. Organization studies, 27(8), 1179-1208. DOI: $10.1177 / 0170840606066312$.

Nonaka, I., \& Toyama, R. (2007). Strategic management as distributed practical wisdom (phronesis). Industrial and Corporate Change, 16(3), 371-394. DOI: 10.1093/icc/dtm014.

Nonaka, I. (2007). The Knowledge-Creating Company. Harvard Business Review, 85, 162-171.

Phelps, C., Heidl, R., \& Wadhwa, A., (2012). Knowledge, networks, and knowledge networks: A review and research agenda. Journal of Management, 38(4), 1115-1166. DOI: 10.1177/0149206311432640.

Rai, R., \& Prakash, A. (2012). A relational perspective to knowledge creation: Role of servant leadership. Journal of Leadership Studies, 6(2), 61-85. DOI: 10.1002/j1s.21238.

Rubinov, M., \& Sporns, O. (2010). Complex network measures of brain connectivity: uses and interpretations. Neuroimage, 52(3),

1059-1069.

DOI: 10.1016/j.neuroimage.2009.10.003.

Schilling, M.A., \& Phelps, C.C. (2007). Interfirm collaboration networks: The impact of largescale network structure on firm innovation. Management Science, 53(7), 1113-1126. DOI: $10.1287 / \mathrm{mnsc} .1060 .0624$.

Singh, J., \& Fleming, L. (2010). Lone inventors as sources of breakthroughs: Myth or reality? Management Science, 56(1), 41-56.

Smith, E.A. (2001). The role of tacit and explicit knowledge in the workplace. Journal of Knowledge Management, 5(4), 311-321. DOI: 10.1108/13673270110411733.

Sosa-García, J., Vega-Pons, S., \& Ruiz-Shulcloper, J. (2012). Algoritmos de agrupamiento difuso, índices de validación: un estado del arte. Reporte técnico Reconocimiento de Patrones. La Habana, Cuba: Centro de Aplicaciones de Tecnologías Avanzadas.

Sutanto, J., Tan, C.H., Battistini, B., \& Phang, C.W. (2011). Emergent leadership in virtual collaboration settings: A social network analysis approach. Long Range Planning, 44(5), 421-439. DOI: 10.1016/j.lrp.2011.09.001.

Takeuchi, H. (2001). Towards a universal management of the concept of knowledge. En Nonaka, I. \& Teece, D. (eds.) (2001), Managing Industrial Knowledge: Creation, Transfer and Utilization, London: Sage

Takeuchi, H. (2013). Knowledge-based view of strategy. Universia Business Review, 40, 68-79. 
Wang, M.C., \& Fang, S.C. (2011, June). Accumulation of knowledge capabilities: The perspective of knowledge-based view and network theory. In Technology Management Conference (ITMC), 2011 IEEE International (pp. 383-387). IEEE.

Wasserman, S., \& Faust, K. (1994). Social network analysis: Methods and applications (Vol. 8). Cambridge University Press.

Wei, C., \& Alvarenga, R. (2010). Beyond the ba: managing enabling contexts in knowledge organizations. Journal of Knowledge Management, 14(4), 592-610. DOI: 10.1108/13673271011059545.

Yun, S., \& Lee, J. (2013). An innovation network analysis of science clusters in South Korea and Taiwan. Asian Journal of Technology Innovation,21(2), 277-289. DOI: 10.1080/19761597.2013.866310. 